\title{
Structure Inclination Angles in the Convective Atmospheric Surface Layer
}

\author{
Kapil Chauhan • Nicholas Hutchins . \\ Jason Monty · Ivan Marusic
}

Received: date / Accepted: date

\begin{abstract}
Two-point correlations of the fluctuating streamwise velocity are examined in the atmospheric surface layer on the salt flats of Utah's western desert, and corresponding structure inclination angles are obtained for neutral, stable and unstable conditions. The neutral surface-layer results supplement evidence for the invariance of the inclination angle given in Marusic and Heuer (Phys. Rev. Lett., 2007, Vol. 99, 114504). In an extension of those results it is found that the inclination angle changes drastically under different stability conditions in the surface layer, varying systematically with the Monin-Obukhov stability parameter in the unstable regime. The variation is parameterized and subsequently can be used to improve existing near-wall models in the large-eddy simulation of the atmospheric surface layer.
\end{abstract}

Keywords Large-scale coherence - Turbulent boundary layer - Unstable surface layer

\section{Introduction}

Over the past few decades, large-eddy simulations (LES) have provided commendable success in advancing the computation of atmospheric surface-layer (ASL) flows. LES methods for such flows are temporally dynamic and spatially efficient, and with advances in computational resources are becoming state-of-the art (e.g. Porté-Agel et al. 2000; Brasseur and Wei 2010). Large-eddy simulations of the high Reynolds number ASL require near-wall modelling and estimates of sub-filter scale stresses (Porté-Agel et al. 2001; Sullivan et al. 2003; Bou-Zeid et al. 2005). Furthermore, the prescribed surface boundary conditions are critical for the performance of LES. In simulations, the first grid point in the LES model is far away from the surface in terms of the viscous scaled wall length and is typically in the logarithmic layer well above the viscous sublayer. However, the near-wall region is responsible for the highest levels of turbulent kinetic energy (TKE) production.

Department of Mechanical Engineering, The University of Melbourne, Parkville 3010, Victoria, Australia

*E-mail: kchauhan@unimelb.edu.au 
This characteristic of wall-bounded flows requires replacing the common no-slip boundary condition with a condition on the wall shear stress. Schumann (1975) proposed one of the first models to directly relate the shear stress at the wall to the velocity at a certain height $z$ above from the wall.

$$
\tau_{i 3}(x, y, t)=\frac{\langle\tau\rangle}{\langle\tilde{u}(z)\rangle} \tilde{u}_{i}(x, y, z, t), \quad(i=1,2) .
$$

Here, the angular brackets \langle\rangle indicate an averaging operation over the homogenous horizontal plane and $\left(^{\sim}\right)$ denotes the spatial filtering operation. Thereby, $\langle\tilde{u}(z)\rangle$ is the mean horizontal velocity at height $z$ and $\tilde{u}_{i}(x, y, z, t)$ is the instantaneous (filtered over the grid size) velocity in the $i$-direction. The mean shear stress $\langle\tau\rangle$ is typically calculated from the average velocity $\langle\tilde{u}(z)\rangle$ based on the law of the wall in the case of the neutral surface layer, or a drag rule based on Monin-Obukhov similarity (Moeng 1984). Grötzbach (1987) successfully used a similar approach to model the wall heat flux and Eq. 1 is commonly referred as the SchumannGrötzbach (SG) model. In an alternate approach that is also commonly used in LES, the total surface shear stress is estimated by applying the Monin-Obukhov similarity for the mean flow to the total instantaneous velocity in the homogenous plane (Moeng 1984; Schmidt and Schumann 1989; Albertson and Parlange 1999). An alternative form of Eq. 1 is the shifted Schumann-Grötzbach (shifted SG) model (Piomelli et al. 1989) wherein the instantaneous filtered velocity at a shifted streamwise location is utilized as,

$$
\tau_{i 3}(x, y, t)=\frac{\langle\tau\rangle}{\langle\tilde{u}(z)\rangle} \tilde{u}_{i}(x+\Delta x, y, z, t), \quad(i=1,2),
$$

where $\Delta x$ is a streamwise displacement. The shifted SG model provides an improvement over the standard SG model (e.g. Piomelli et al. 1989; Porté-Agel et al. 2000; Piomelli and Balaras 2002) and has been extensively evaluated (Marusic et al. 2001; Venugopal et al. 2003; Chamorro and Porté-Agel 2010). Another model proposed by Marusic et al. (2001) is derived from wind-tunnel experiments that compared directly measured surface shear stress to the modelled stress. The Marusic et al. (2001) model is based on the streamwise velocity and given as,

$$
\tau_{i 3}(x, y, t)=\frac{\langle\tau\rangle}{\langle\tilde{u}(z)\rangle} \tilde{u}_{i}(x, y, z, t)-\alpha U_{\tau}\left[\tilde{u}_{i}(x+\Delta x, y, z, t)-\left\langle\tilde{u}_{i}(z)\right\rangle\right], \quad(i=1,2),
$$

where $\alpha=0.1$ is a characteristic constant and $U_{\tau}$ is the mean friction velocity. The model of Marusic et al. (2001) was formulated to correctly estimate the energy levels for the fluctuating wall shear stress such that the resulting spectra at different positions from the wall collapse onto a single curve. Experimental studies (e.g. Carper and Porté-Agel 2004; Marusic and Heuer 2007; Li and Bou-Zeid 2011) are helpful in undertaking an a priori evaluation of the wall models, wherein the agreement of the prediction from a particular model with the measurements forms the basis of its validity. On the other hand, in an a posteriori study the wall models are implemented in LES and the results are evaluated for agreement with standard boundary-layer theory (e.g. Stoll and Porté-Agel 2006, 2008). In all of the models mentioned above the streamwise shift $\Delta x$ plays a vital role in accurately estimating the instantaneous surface shear stress. 
The idea behind using a streamwise shift $\Delta x$ arises from the fact that inclined vortical features in the near-wall region of wall-bounded flows are significantly responsible for energy transfer and crucial to forward-scatter and backscatter in sub-filter-scale dissipation in large-eddy simulations (Carper and Porté-Agel 2004). Events like ejection and sweep and the recently documented 'superstructures' (Hutchins and Marusic 2007; Marusic et al. 2010) result in a strong correlation between the local velocity fluctuations and the fluctuating surface shear stress. Such coherent structures have been observed experimentally in both instantaneous flow fields and using conditional averaging and two-point correlations (Brown and Thomas 1977; Head and Bandyopadhyay 1981; Adrian et al. 2000). The presence of these coherent motions has also been reported in the atmospheric surface layer (Hommema and Adrian 2003; Carper and Porté-Agel 2004; Morris et al. 2007; Hutchins et al. 2012). The presence of such inclined instantaneous motions, occurring frequently at a multitude of scales, results in two-point correlation maps that are elliptical in shape with the principal axis inclined at a shallow angle away from the wall. This angle is often referred to as the structure inclination angle. The peak of correlation occurs at a streamwise shift $\Delta x^{*}$ (Eq. 2) that in the neutral surface layer typically corresponds to an inclination angle of approximately $14^{\circ}$ (Carper and Porté-Agel 2004; Marusic and Heuer 2007). Marusic and Heuer (2007) demonstrated the invariance of the inclination angle in wall-bounded flows with zero buoyancy (neutral conditions) over a wide range of Reynolds number through laboratory and field experiments. However, the study by Marusic and Heuer (2007) does not document the temporal lag or shift between velocity and shear stress in non-neutral conditions. In this paper we study the change in the inclination angle (directly related to the streamwise shift $\Delta x^{*}$ ) for varying stability conditions, where stability is characterized by the Monin-Obukhov parameter.

The lead-lag is also an important characteristic of the amplitude modulation of small-scale turbulence near the surface by the large-scale motions away from the wall (Mathis et al. 2009). As such, the structure inclination angle is also an important parameter in the predictive model of Marusic et al. (2010, 2011) where the statistics of streamwise shear stress and velocity fluctuations near the surface can be predicted from measurements in the logarithmic region of a neutral surface layer. Although this angle is associated with the presence of inclined structures, the physical mechanism that effectively sets its value between $12^{\circ}-14^{\circ}$ in neutral conditions is unknown. In the neutral surface layer or in laboratory flows without buoyant conditions, the inclination angle found from temporal measurements utilizing Taylor's hypothesis (e.g. Kovasznay et al. 1970; Boppe and Neu 1995; Carper and Porté-Agel 2004; Hutchins et al. 2012) is in agreement with that inferred from direct spatial measurements (e.g. Morris et al. 2007; Ganapathisubramani et al. 2005; Christensen 2001). Variation in the inclination angle under non-neutral conditions has previously been observed; e.g. Phong-Anant et al. (1980) observed 'ramps' in the temperature profiles that were inclined between $21^{\circ}-47^{\circ}$; Hommema and Adrian (2003) visualized large-scale ramp-like structures with downstream inclination of $3^{\circ}-35^{\circ}$; and Carper and Porté-Agel (2004) found an increased inclination angle between $15^{\circ}-35^{\circ}$ under unstable conditions from temporal velocity measurements. The previous results of varying inclination angle in the non-neutral surface layer however are quite sparse and lack characterization with a suitable flow parameter. We address this aspect here by examining the structure inclination angle and its variation with changes in the surface heat 
VIEW NORTH

spanwise array CSAT

- tower CSAT

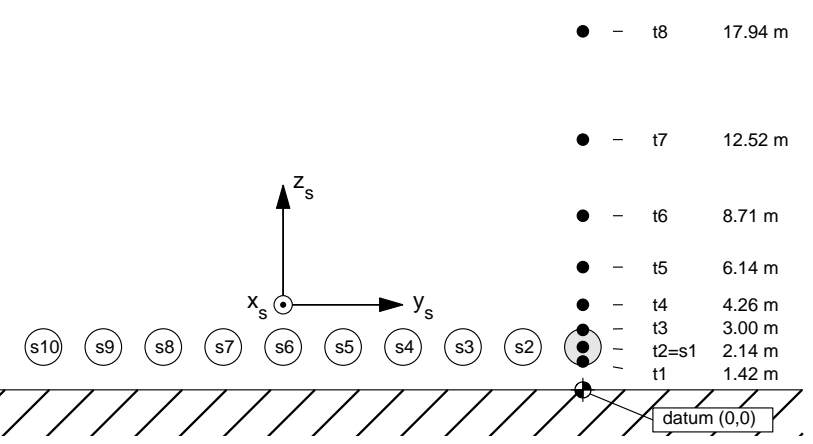

Fig. 1 North view of the sonic anemometers arranged in the spanwise and tower array at the SLTEST site. Note that $s 1=\mathrm{t} 2$. Reproduced from Hutchins et al. (2012)

flux; i.e. stable, unstable and neutral conditions from a larger database of field experiment data (spanning almost nine days of continuous acquisition).

\section{Experimental Set-up}

The results presented are from data acquired by Marusic and Heuer (2007) and Marusic and Hutchins (2008) at the Surface Layer Turbulence and Environmental Science Test (SLTEST) facility in the salt flats of western Utah. The experiments were conducted at the SLTEST site over a duration of nine days from 26 May 2005 to 3 June 2005. Continuous measurements of all three components of velocity and of temperature were acquired by a spanwise and wall-normal array of 18 sonic anemometers (Campbell Scientific CSAT3) in an 'L' shaped configuration. The schematic of the set-up as viewed from the north direction is shown in Fig. 1. The spanwise array covered an overall distance of $27 \mathrm{~m}$ with 10 anemometers placed $3 \mathrm{~m}$ apart and at a height of $z_{\mathrm{s}}=2.14 \mathrm{~m}$. The height of the spanwise array and the average measurement from the 10 sonics is used to determine the MoninObukhov similarity parameter. The wall-normal array consisted of nine probes placed logarithmically from $z_{\mathrm{t}}=1.42$ to $25.69 \mathrm{~m}$. The sonics in the spanwise array are denoted with the prefix ' $s$ ' and the prefix ' $t$ ' is assigned to the tower array. The two-point correlation results presented here are derived from the tower array only. Not all of the data are suitable for analysis after pre-processing to correct for wind direction, de-trending and the application of data selection criteria, details of which are provided in Hutchins et al. (2012). After applying the pre-processing, only 63 hours of data are subsequently analyzed.

\section{Results}

In the study of Marusic and Heuer (2007), the structure inclination angle was obtained by cross-correlation of surface wall shear stress velocity fluctuations $u_{\tau}$ with 
streamwise velocity fluctuations $u$ in the logarithmic region. For the current data, the wall shear stress was not measured and hence the cross-correlation is computed between the sonic anemometer closest to the surface (t1) and the rest of the tower anemometers. As shown in Marusic and Heuer (2007), this procedure gives slightly smaller values of correlation but the calculated angle is not significantly altered if $\Delta z$ is large ( $\Delta z$ is relative height from $\mathrm{t} 1$ ). The cross-correlation of the $u$ signal from $\mathrm{t} 1$ with the $u$ signal from the rest of the tower sonics is plotted in Fig. 2 for $1 \mathrm{hr}$ of neutral surface-layer data. The abscissa on Fig. 2 is shown in length units by converting the temporal lead/lag using Taylor's hypothesis ( $\Delta x=U \Delta t, U$ is the convection velocity taken as the local mean). The cross-correlation is defined as,

$$
R_{u u}(\Delta x, \Delta z)=\frac{\langle u(x, y, z) u(x+\Delta x, y, z+\Delta z)\rangle}{\sigma_{u(x, y, z)} \sigma_{u(x+\Delta x, y, z+\Delta z)}} .
$$

It is seen in Fig. 2 that the signal from $\mathrm{t} 1(z=1.42 \mathrm{~m})$ is highly correlated with the signal from $\mathrm{t} 2(z=2.14 \mathrm{~m})$ with a peak correlation magnitude of approximately 0.7. Further from the wall, the peak correlation magnitude decreases, shown by the sequence of lines in Fig. 2 (peak $R_{u u} \approx 0.1$ between $t 1$ and $\mathrm{t} 9$ ). A shift in the peak location away from $\Delta x=0$ is also observed with decreasing magnitude. This shift (denoted by $\Delta x^{*}$ ) becomes larger as $\Delta z$ increases and the variation of $\Delta x^{*}$ with $\Delta z$ is found to be linear (see inset in Fig. 2). The average structure inclination angle $\gamma$ is calculated as,

$$
\gamma=\arctan \left(\left\langle\Delta z / \Delta x^{*}\right\rangle\right)
$$

The data in Fig. 2 represent the neutral surface layer which has been extensively examined in Hutchins et al. (2012) to report its similarity with canonical laboratory flow. We now look at the structure inclination angle $\gamma$ under flow conditions that are non-neutral; i.e. varying stability. For comparison, the stability parameter adopted here is the Monin-Obukhov parameter $z_{\mathrm{s}} / L$, where $L$ is defined as,

$$
L=-\frac{\bar{\theta} u_{*}^{3}}{\kappa g(\overline{w \theta})_{0}} .
$$

Here, $L$ is the Obukhov length scale determined from the average of 10 sonic anemometers in the spanwise array, $\kappa=0.41$ is the von Kármán constant, $g$ is the gravitational acceleration, $u_{*}=(-\overline{u w})^{1 / 2}$ is the friction velocity, $(\overline{w \theta})_{0}$ is the kinematic surface heat flux, and $\bar{\theta}$ is the mean temperature ${ }^{1}$. The stability parameter is obtained at the reference location $z_{\mathrm{s}}=2.14 \mathrm{~m}$ where the horizontal sonic anemometers were placed. For the present set of data the stability parameter $z / L$ varied between -5 (unstable) to 0.1 (stable), although most hourly flow conditions had positive surface heat flux. To compare $\gamma$ for different stability conditions, $R_{u u}$ is represented in the form of a two-dimensional correlation map in the $x-z$ plane in Figs. 3a - 3c along with a schematic that illustrates the qualitative differences for different stability regimes.

The correlation map for the neutral surface layer $\left(z_{\mathrm{s}} / L \approx 0\right)$ is shown in Fig. 3a. The location of sonic anemometers is indicated for the reader's reference and the condition point is at $\mathrm{t} 1$ (shown in red). It is clear that the streamwise/spanwise

\footnotetext{
1 The difference between air temperature $\theta$ and virtual temperature $\theta_{v}$ due to moisture is small and can be ignored for the ASL over land (Kaimal and Finnigan 1994).
} 


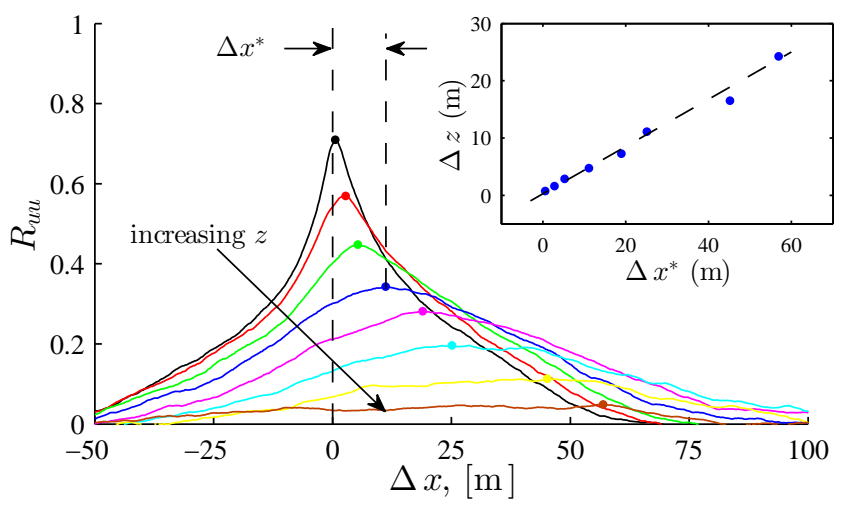

Fig. 2 Cross-correlation, $R_{u u}$ at different heights with $u_{\mathrm{t} 1}$ as the reference signal. ' $\bullet$ ' indicates peak $R_{u u}$ between $\mathrm{t} 1$ and $\mathrm{t} 2-\mathrm{t} 8$. Insert shows $\Delta z$ vs. $\Delta x^{*}$ for the eight $R_{u u}$ profiles and its linear trend.

$R_{u u}$ map has a large associated wall-normal extent; i.e. positive $R_{u u}$ extends beyond the top of the tower. The region of positive correlation is high and extremely long in the streamwise direction (extending beyond the limits shown). The positive contours have a clear inclination in the streamwise direction, as previously documented in laboratory flows (e.g. Kovasznay et al. 1970; Brown and Thomas 1977; Christensen and Adrian 2001) and also in the atmospheric surface layer (Carper and Porté-Agel 2004; Marusic and Heuer 2007). The structure inclination angle for the neutral surface layer in Fig. $3 \mathrm{a}$ is $13.7^{\circ}$ indicated by the solid line. Marusic and Heuer (2007) reported $\gamma=14.7^{\circ}$ from $R_{u_{\tau} u}$ (correlation of $u_{\tau}$ and $u$ ) and $\gamma=11.8^{\circ}$ for $R_{u u}$ for the neutral surface layer at the same facility. Within the experimental scatter, our values for the neutral surface layer agree well with the recent experiments of Marusic and Heuer (2007) and those reported in earlier studies $\left(\gamma=18^{\circ}\right.$ from Brown and Thomas (1977), $\gamma=15^{\circ}$ from Boppe et al. (1999), and $\gamma=16^{\circ}$ from Carper and Porté-Agel (2004)).

Figure $3 \mathrm{~b}$ shows the $R_{u \text { u }}$ map for an hour of unstable surface-layer data where $z_{\mathrm{s}} / L \approx-0.1$ is encountered. Again, there is a large region of positive correlation that extends beyond the measurement array in the wall-normal direction and is significantly elongated in the streamwise direction. Similar to the neutral case, the $R_{u u}$ map has a distinct inclination, however the angle of inclination is clearly higher than for the neutral case $3 \mathrm{a}$. For this hour of data the structure inclination angle is $34.3^{\circ}$, which is more than twice that of the neutral case. The increase in inclination angle up to $34^{\circ}$ with decreasing stability has also been reported previously by Carper and Porté-Agel (2004). It appears that the unstable surface layer has a larger spatial extent of meaningful correlation and hence the underlying coherence occurs at a larger physical scale.

A plausible explanation for the increased inclination angle in unstable surface layers provided by Hommema and Adrian (2003) is the superposition of sheardriven coherent flow features and buoyancy driven motions.During the day, when $\mathrm{d} \theta / \mathrm{d} z<0$, the total production of turbulence is augmented by the additional contribution by positive buoyancy along with the shear-driven production of turbulence. The spatial extent of the two-point correlation represents the signature 

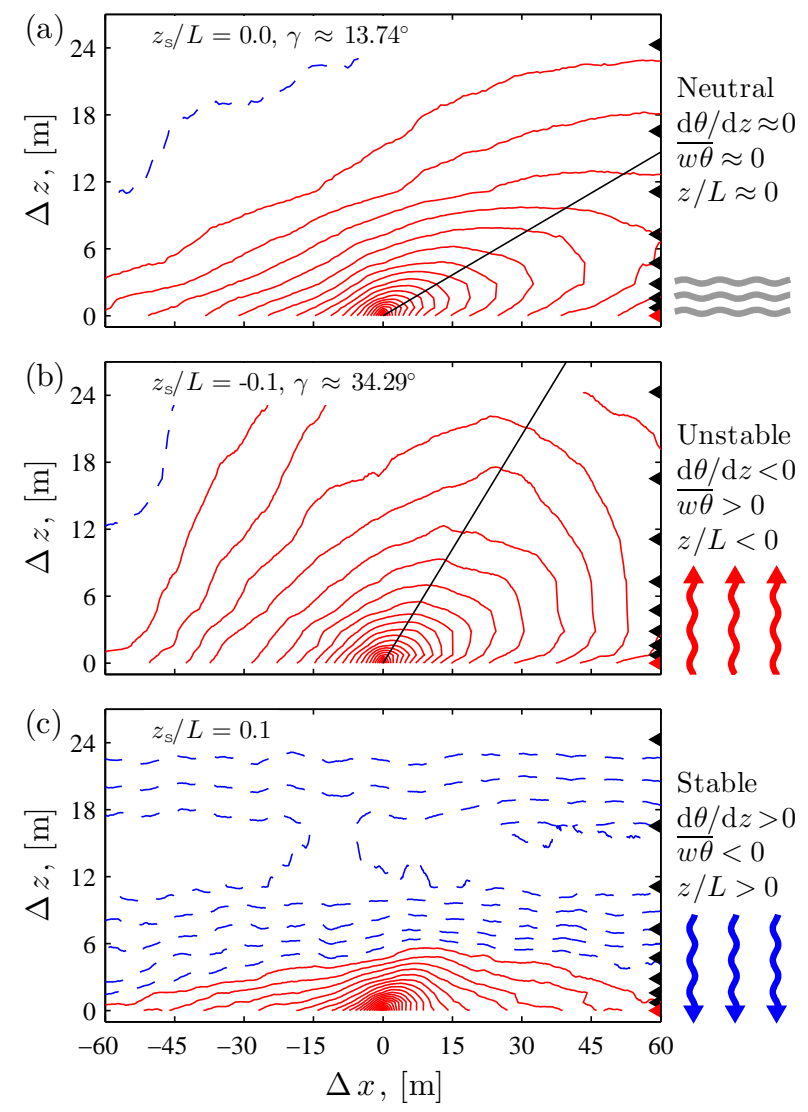

Fig. $3 R_{u u}$ correlation map in the $x-z$ plane constructed from the tower array. Solid line indicates the inclination of $R_{u u}$. (a) Neutral, (b) Unstable, and (c) Stable. Contour levels are from $R_{u u}=-0.15$ to 0.95 in increments of 0.05 . Solid red lines indicate positive contour levels and dashed blue line indicate negative levels. Note that data for cases (a), (b) and (c) were acquired on different days.

of large-scale features/motions that exist in the boundary layer (Ganapathisubramani et al. 2003; Hutchins and Marusic 2007). These motions are streamwise aligned and are present in a region that is very close to the wall and extends up to the outer part of the logarithmic layer. Buoyancy in the atmospheric surface layer essentially represents a force that acts normal to the preferred orientation of these structures. Wall-normal motions induced or augmented by buoyancy can become of the same order of magnitude as these large structures, and consequently alter their orientation. Thus, buoyancy can be interpreted to have a 'lifting' effect resulting in the increased inclination angle of the structures (Carper and Porté-Agel 2004). Similar spatial changes in the structure of $R_{u u}$ in the streamwise/spanwise plane were observed by Chauhan et al. (2010), where the iso-contours are elongated and become wider as the heat flux $\overline{w \theta}$ increases. Under near-neutral conditions the effects of buoyancy diminish and the heat flux decreases to near zero resulting in predominantly shear-driven turbulence throughout the surface layer. 


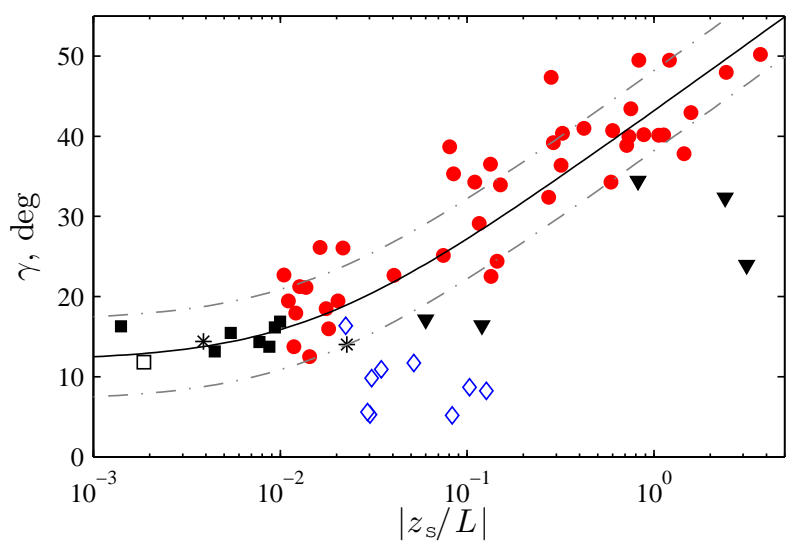

Fig. 4 Variation of structure inclination angle $\gamma$ with the stability parameter $z_{\mathrm{s}} / L$. Solid line represents Eq. 7. The neutral surface-layer data is categorized as $\left|z_{\mathrm{s}} / L\right|<0.01$ and shown by ' $\square$ '. ' $\bullet$ ' indicates unstable surface-layer data and ' $\diamond$ ' indicates stable surface-layer data. The statistics for each data point are obtained from one hour of data. '*', from $R_{u_{\tau} u}$ of Marusic and Heuer (2007); ' $\square$ ', from $R_{u u}$ of Marusic and Heuer (2007) $(z / L$ at $z=2.93 \mathrm{~m})$; ' $\boldsymbol{\nabla}$ ', from Carper and Porté-Agel (2004) $(z / L$ at $z=4.9 \mathrm{~m})$. Dash-dotted lines indicate $\pm 5^{\circ}$ deviation from Eq. 7 .

In Fig. 3c the correlation map for an hour of stable surface-layer data with $z_{\mathrm{s}} / L=0.1$ is plotted. Note that the absolute value of $z_{\mathrm{s}} / L$ (denoted as $\left|z_{\mathrm{s}} / L\right|$ ) is the same in Figs. 3b and 3c. The characteristics of $R_{u u}$ for the stable case are distinctively different to the unstable case. Near the surface the region of positive correlation still appears to be long although the magnitude of correlation is smaller at a particular $\Delta x$ indicating that coherent structures are shortened in $x$. Consistent with the smaller surface-layer depth at night, it is noticeable that the wall-normal extent of the positive correlation is now significantly smaller compared to the neutral and unstable cases. A large region of negative correlation exists, occupying more than $3 / 4$ the wall-normal height of the tower. These features indicate that under stable conditions the underlying coherent structure is clearly very different. The almost parallel contour lines also indicate that the $R_{u u}$ map lacks the physical signature of vortical features that are common to Figs. 3a and 3b. Although locally $R_{u u}$ seems to be inclined very close to the surface implying such vortical features might be present at a smaller scale close to the surface. As the temperature decreases at sunset, the effects of buoyancy diminish and it is common for the heat flux to become negative at night time. This leads to negative buoyancy and a positive thermal gradient $(\mathrm{d} \theta / \mathrm{d} z>0)$, in which turbulent motions are suppressed by gravity. It is reasoned that under stable conditions density stratification near the surface leads to a 'layered' flow. The cold and denser air near the surface restricts transport or momentum transfer that is typically associated with wall-turbulence mechanisms. Subsequently the shear-induced turbulence is only dominant within a limited wall-normal extent beyond which the shear production diminishes.

Figure 4 plots the change in inclination angle $\gamma$ obtained from the $R_{u u}$ maps versus the stability parameter $z_{\mathrm{s}} / L$ for all the hourly data. As expected, the inclination angle increases with decreasing stability (increasing $-z_{\mathrm{s}} / L$ ). The variation 
of $\gamma$ with $\left|z_{\mathrm{s}} / L\right|$ is systematic and follows a log-linear increase in the unstable regime. For the few hourly cases of stable conditions that existed in these measurements the inclination angles are reduced (typically below $10^{\circ}$ ) and lack an overall trend (these are shown by the diamond symbols on Fig. 4). Given that the region of positive correlation near the surface is quite small and only four sonic anemometers were measuring below $6 \mathrm{~m}$ (see Fig. 3c), the $\gamma$ for the stable regime should be considered as a qualitative indicator. For comparison the inclination angles calculated by Marusic and Heuer (2007) and Carper and Porté-Agel (2004) from field experiments are also included in the figure. The stability parameter in the study of Carper and Porté-Agel (2004) was obtained at $z=4.9 \mathrm{~m}$, which is a plausible reason for their data to appear shifted in comparison to the trend in the present study. It should be noted that this atmospheric surface-layer data are not well-converged and the scatter in Fig. 4 would be smaller in a controlled laboratory experiment. A parametric equation is fitted to the log-linear trend of unstable data to model the variation of $\gamma$ with $z_{\mathrm{s}} / L$ and is given as,

$$
\gamma=\gamma_{0}+7.3 \ln \left(1-70 z_{\mathrm{s}} / L\right)
$$

where $z_{\mathrm{s}}=2.14 \mathrm{~m}$ and $\gamma_{0}=12^{\circ}$ is the inclination angle in the neutral surface layer. The functional form of Eq. 7 approaches the invariant value of inclination angle when $z_{\mathrm{s}} / L$ approaches zero, while it depicts the logarithmic behaviour of $\gamma$ at high $-z_{\mathrm{s}} / L$. In our study, the Monin-Obukhov parameter is estimated from the 10 sonic anemometers at $z_{\mathrm{s}}=2.14 \mathrm{~m}$ to obtain a reliable average estimate of $z / L$. We find that the trend of $\gamma$ versus $z / L$ is not significantly altered and the scatter is within $\pm 5^{\circ}$ of Eq. 7 if a different reference height of $z=1.42 \mathrm{~m}$ or $z=3 \mathrm{~m}$ is used instead of $z_{\mathrm{s}}=2.14 \mathrm{~m}$ on the abscissa of Fig. 4 . Therefore, the variation of the average inclination angle could also be characterized by $z / L$ from a different height that provides a good measure of stability near the surface. It should be noted that Eq. 7 would not be applicable in the unstable ASL with very large $-z_{\mathrm{s}} / L$ where the flow approaches free convection. In such cases the inclination angle would no longer be a physical characteristic of the underlying motions (e.g. thermal plumes). Equation 7 agrees well with the current data and also with the values previously reported (Carper and Porté-Agel 2004; Marusic and Heuer 2007).

In summary, a systematic variation of the structure inclination angle with the Monin-Obukhov similarity parameter is documented in this study. Large-scale coherent motions such as the hairpin vortex packets are typically present across the entire surface layer and contribute significantly to $R_{u u}$. Further, Hommema and Adrian (2003) postulated that the structure inclination angle in unstable surface layers results from the superposition of hairpin vortex packets and a buoyant upward motion due to thermal stratification. With such a perspective we can infer that the deviation of the inclination angle from the invariant value of the neutral surface layer is solely caused by the thermal effects and hence should be characterized by the stability parameter.

\section{References}

Adrian RJ, Meinhart CD, Tomkins CD (2000) Vortex organization in the outer region of the turbulent boundary layer. J Fluid Mech 422:1-54

Albertson JD, Parlange MB, Surface length scales and shear stress: Implications for landatmosphere interaction over complex terrain. Water Resour Res 35(7):2121-2132 
Boppe RS, Neu WL (1995) Quasi-coherent structures in the marine atmospheric surface layer. J Geophys Res 100(C10):20635-20648

Boppe RS, Neu WL, Shuai H (1999) Large-scale motions in the marine atmospheric surface layer. Boundary-Layer Meteorol 92(2):165-183

Bou-Zeid E, Meneveau C, Parlange MB (2005) A scale-dependent Lagrangian dynamic model for the large-eddy simulation of complex turbulent flows. Phys Fluids 17:025105

Brasseur J, Wei T (2010) Designing large-eddy simulation of the turbulent boundary layer to capture law-of-the-wall scaling. Phys Fluids 22:021303

Brown GL, Thomas ASW (1977) Large structure in a turbulent boundary layer. Phys Fluids 20(10):243-252

Carper MA, Porté-Agel F (2004) The role of coherent structures in subfilter-scale dissipation of turbulence measured in the atmospheric surface layer. J Turbul 5(40)

Chamorro L, Porté-Agel F (2010) Wind-tunnel study of surface boundary conditions for largeeddy simulation of turbulent flow past a rough-to-smooth surface transition. J Turbul 11(1):1-17

Chauhan K, Hutchins N, Marusic I, Monty J (2010) Two-point correlation statistics in atmospheric surface layers. In: Proc. 17th Australasian Fluid Mech Conf, Paper no. 141

Christensen KT (2001) Experimental investigation of acceleration and velocity fields in turbulent channel flow. PhD thesis, University of Illinois, USA.

Christensen KT, Adrian RJ (2001) Statistical evidence of hairpin vortex packets in wall turbulence. J Fluid Mech 431:433-443

Ganapathisubramani B, Longmire EK, Marusic I (2003) Characteristics of vortex packets in turbulent boundary layers. J Fluid Mech 478:35-46

Ganapathisubramani B, Hutchins N, Hambleton WT, Longmire EK, Marusic I (2005) Investigation of large-scale coherence in a turbulent boundary layer using two-point correlations. J Fluid Mech 524:57-80

Grötzbach G (1987) Direct numerical and large eddy simmulations of turbulent channel flows. In: Cheremisinoff NP (ed) Encyclopedia of Fluid Mechanics, Vol 6, Gulf, West Orange, NJ, pp 1337-1391

Head MR, Bandyopadhyay P (1981) New aspects of turbulent boundary-layer structure. J Fluid Mech 107:297-337

Hommema S, Adrian RJ (2003) Packet structure of surface eddies in the atmospheric boundary layer. Boundary-Layer Meteorol 106:147-170

Hutchins N, Marusic I (2007) Evidence of very long meandering streamwise structures in the logarithmic region of turbulent boundary layers. J Fluid Mech 579:1-28

Hutchins N, Chauhan K, Marusic I, Monty J, Klewicki J (2012) Towards reconciling the structure of boundary layers in the atmosphere and laboratory. Boundary-Layer Meteorol 145:273-306 (DOI 10.1007/s10546-012-9735-4)

Kaimal JC, Finnigan JJ (1994) Atmospheric Boundary Layer Flows: Their Structure and Measurement. Oxford University Press, New York, 289 pp.

Kovasznay LSG, Kibens V, Blackwelder RF (1970) Large-scale motion in the intermittent region of a turbulent boundary layer. J Fluid Mech 41:283-326

Li D, Bou-Zeid E (2011) Coherent structures and the dissimilarity of turbulent transport of momentum and scalars in the unstable atmospheric surface layer. Boundary-Layer Meteorol 140:243-262

Marusic I, Heuer W (2007) Reynolds number invariance of the structure inclination angle in wall turbulence. Phys Rev Lett 99:114504

Marusic I, Hutchins N (2008) Study of the log-layer structure in wall turbulence over a very large range of Reynolds number. Flow Turbul Combust 81:115-130

Marusic I, Kunkel G, Porté-Agel F (2001) Experimental study of wall boundary conditions for large-eddy simulation. J Fluid Mech 446:309-320

Marusic I, Mathis R, Hutchins N (2010) Predictive model for wall-bounded turbulent flow. Science 9:193-196

Marusic I, Mathis R, Hutchins N (2011) A wall-shear stress predictive model. In: J Phys Conference Series - Proceedings of the $13^{\text {th }}$ European Turbulence Conference, Warsaw, Poland 318:012003

Mathis R, Hutchins N, Marusic I (2009) Large-scale amplitude modulation of the small-scale structures of turbulent boundary layers. J Fluid Mech 628:311-337

Moeng CH (1984) A large-eddy simulation for the study of planetary boundary layer turbulence. J Atmos Sci 41(13):2052-2062 
Morris SC, Stolpa SR, Slaboch PE, Klewicki JC (2007) Near-surface particle image velocimetry measurements in a transitionally rough-wall atmospheric boundary layer. J Fluid Mech 580:319-338

Phong-Anant D, Antonia RA, Chambers AJ, Rajagopalan S (1980) Features of the organized motion in the atmospheric surface layer. J Geophys Res 85(C1):424-432

Piomelli U, Balaras E (2002) Wall-layer models for large-eddy simulations. Ann Rev Fluid Mech 34:349-374

Piomelli U, Ferziger J, Moin P, Kim J (1989) New approximate boundary conditions for large eddy simulations of wall bounded flows. Phys Fluids 1:1061-1068

Porté-Agel F, Meneveau C, Parlange MB (2000) A scale-dependent dynamic model for largeeddy simulation: application to a neutral atmospheric boundary layer. J Fluid Mech 415:261-284

Porté-Agel F, Parlange MB, Meneveau C, Eichinger WE (2001) A priori field study of the subgrid-scale heat fluxes and dissipation in the atmospheric surface layer. J Atmos Sci 58:2673-2698

Schmidt H, Schumann U (1989) Coherent structure of the convective boundary layer derived from large-eddy simulations. J Fluid Mech 200:511-562

Schumann U (1975) Subgrid scale model for finite difference simulations of turbulent flows in plane channels and annuli. J Comput Phys 18:376-404

Stoll R, Porté-Agel F (2006) Effect of roughness on surface boundary conditions for large-eddy simulation. Boundary-Layer Meteorol 118:169-187

Stoll R, Porté-Agel F (2008) Large-eddy simulation of the stable atmospheric boundary layer using dynamic models with different averaging schemes. Boundary-Layer Meteorol 126:128

Sullivan PB, Horst TW, Lenschow DH, Moeng CH, Weil JC (2003) Structure of subfilter-scale fluxes in the atmospheric surface layer with application to large-eddy simulation modelling. J Fluid Mech 482:101-139

Venugopal V, Porté-Agel F, Foufoula-Georgiou E, Carper M (2003) Multiscale interactions between surface shear stress and velocity in turbulent boundary layers. J Geophys Res 108:4613 


\section{University Library}

\section{- M M I N E R VA A gateway to Melbourne's research publications}

Minerva Access is the Institutional Repository of The University of Melbourne

Author/s:

Chauhan, K;Hutchins, N;Monty, J;Marusic, I

Title:

Structure Inclination Angles in the Convective Atmospheric Surface Layer

Date:

2013-04-01

Citation:

Chauhan, K., Hutchins, N., Monty, J. \& Marusic, I. (2013). Structure Inclination Angles in the Convective Atmospheric Surface Layer. BOUNDARY-LAYER METEOROLOGY, 147 (1), pp.41-50. https://doi.org/10.1007/s10546-012-9777-7.

Persistent Link:

http://hdl.handle.net/11343/282678 